\title{
When GENErosity backfires
}

bacteria
represent a
reservoir of
antibacterial
genes that can
be co-opted
for function in
other domains
of life

Horizontal gene transfer (HGT) occurs most frequently among closely related organisms and facilitates the acquisition of adaptive traits. There are relatively few reports of HGT across phylogenetic domains, but two new studies now describe inter-kingdom HGT of two distinct families of bacterial genes, both of which encode antibacterial proteins, and show that these proteins are functional in their eukaryotic and archaeal hosts.

Mougous and colleagues assessed the phylogenetic distribution of the bacterial type VI secretion amidase effector (tae) genes, which encode four families of peptidoglycandegrading toxins that function in interbacterial antagonism. Each Tae family displays substrate specificity: members of families 1

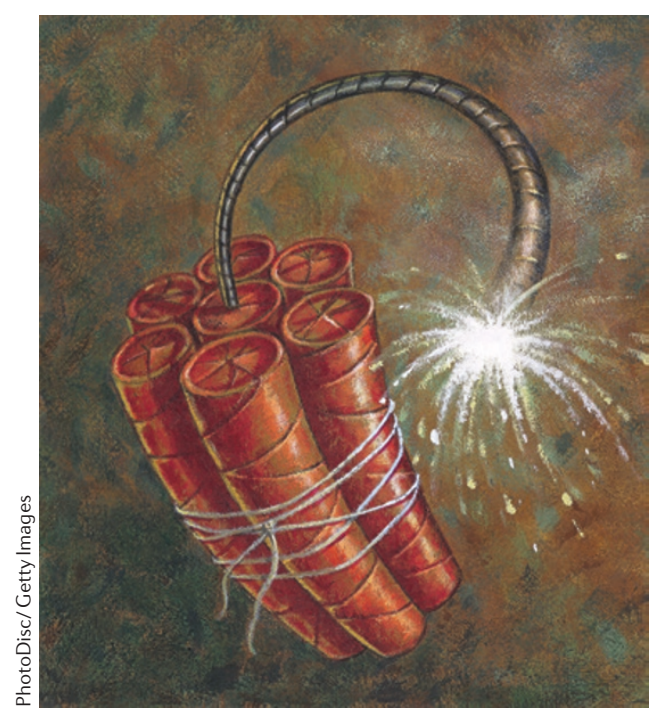

and 4 cleave the $\gamma$-D-glutamyl-mesodiaminopimelic acid (mDAP) bond, whereas those from families 2 and 3 cleave the mDAP-D-alanine crosslinking bond. Interestingly, the authors found that three of the four tae gene families (tae 2-4) have homologues in diverse eukaryotic genomes and have been transferred to eukaryotes on at least six different occasions, resulting in the domesticated amidase effector (dae) genes.

In bacteria, the Tae effectors are secreted by the type VI secretion system in a Sec-independent manner; however, the authors discovered that most of the Dae proteins had acquired eukaryotic Sec signals, which suggests that they have been functionally specialized for export in eukaryotes, thereby providing support for an adaptive function. Indeed, quantitative reverse transcription PCR revealed that the dae genes are expressed in eukaryotes and, remarkably, the Dae enzymes from representative eukaryotic hosts retained the ability to degrade peptidoglycan according to the substrate specificities of their Tae homologues, which suggests that they have been maintained in eukaryotes for their antibacterial function. Consistent with this, the authors found that the Dae2 enzyme of the deer tick Ixodes scapularis was capable of cleaving the peptidoglycan sacculus of the bacterium Borrelia burgdorferi (which causes Lyme disease) in vitro and its expression also restricted the proliferation of B. burgdorferi in vivo.

In the second study, Metcalf et al. examined the phylogenetic distribution and function of a bacterial lysozyme gene, which encodes a glycosyl hydrolase 25 muramidase that also degrades peptidoglycan. The authors observed that this gene was transferred from bacteria to other organisms repeatedly (at least four times), and that homologues are present in several disparate taxa that span the entire tree of life, including a plant, an insect, several fungal species and the deep-sea hydrothermal vent archaeon Aciduliprofundum boonei. The archaeal lysozyme was shown to retain antibacterial activity and during co-culture with the bacterium Mesoaciditoga lauensis (which also inhabits hydrothermal vents), lysozyme expression was upregulated, suggesting that it is induced in response to bacterial competition.

Collectively, these studies emphasize that the inter-kingdom barriers to HGT can be overcome and that bacteria represent a reservoir of antibacterial genes that can be co-opted for function in other domains of life.

Christina Tobin Kåhrström

ORIGINAL RESEARCH PAPERS Chou, S. Daugherty, M. D. et al. Transferred interbacterial antagonism genes augment eukaryotic innate immune function. Nature https://dx.doi.org/ doi:10.1038/nature13965 (2014)| Metcalf, J. A. et al. Antibacterial gene transfer across the tree of life. eLife 3, e04266 (2014) 